\title{
Healthcare improvement science
}

Brigita Skela-Savič, Rhoda Macrae, Manuel Lillo-Crespo, Kevin D Rooney

\section{Source}

Brigita Skela-Savič, Rhoda Macrae, Manuel Lillo-Crespo, Kevin D Rooney. (2017). The

development of a consensus definition for healthcare improvement science (HIS) in

seven European countries: A consensus methods approach.

Healthcare improvement science is the generation of knowledge to cultivate change and deliver person-centred care that is safe, effective, efficient, equitable and timely. It improves patient outcomes, health system performance and population health. 